\title{
Interrelations between migraine-like headache and persistent post-traumatic headache attributed to mild traumatic brain injury: a prospective diary study
}

Håkan Ashina', Afrim Iljazi ${ }^{1}$, Faisal M. Amin ${ }^{1}$, Messoud Ashina ${ }^{1}$, Richard B. Lipton ${ }^{2,3+}$ and Henrik W. Schytz ${ }^{1 *+}$

\begin{abstract}
Background: Persistent post-traumatic headache (PTH) is a common sequela of mild traumatic brain injury (TBI) and retrospective assessments have found a migraine-like phenotype to be very frequent. This has raised a discussion of shared underlying mechanisms and whether persistent PTH is simply trauma-triggered migraine.

Methods: A 28-day prospective diary study with daily entries and acquisition of data on headache characteristics, associated symptoms, and acute medication use. A total of 64 patients with persistent PTH were enrolled from April 2019 to August 2019. Outcomes were the proportion of monthly headache days of any intensity that met the criteria for a migraine-like day or TH-like day, as well as the corresponding figures for monthly headache days of moderate to severe intensity. Headache phenotypes were initially assigned based on diagnostic evaluation by semistructured interview, whilst final headache phenotypes were assigned by diary review.

Results: After diary review, we found that monthly headache days were exclusively migraine-like in 24 of 64 patients (38\%) and exclusively TTH-like days in 8 of 64 patients (13\%). Considering only monthly headache days of moderate to severe intensity, the corresponding figures were 35 of 64 patients (55\%) for migraine-like days and 8 of 64 patients (13\%) for TTH-like days. The following headache phenotypes were assigned based on diary review: chronic migraine-like $(n=47,73 \%)$, combined episodic migraine-like and chronic TTH-like $(n=9,13 \%)$, and 'pure' chronic TTH-like $(n=8,13 \%)$.

Conclusions: A migraine-like phenotype is common in patients most adversely affected by persistent PTH, although some patients did have a pure chronic THH-like phenotype. At minimum, these findings suggest that persistent PTH is - at least in some - not 'trauma-triggered migraine'.
\end{abstract}

Keywords: Concussion, Head trauma, Diagnosis, Clinical characteristics

\footnotetext{
* Correspondence: henrik.winther.schytz.01@regionh.dk

${ }^{\dagger}$ Richard B. Lipton and Henrik W. Schytz contributed equally to this work. 'Danish Headache Center, Department of Neurology, Rigshospitalet Glostrup, Faculty of Health and Medical Sciences, University of Copenhagen, Valdemar Hansen Vej 5, Glostrup, DK-2600 Copenhagen, Denmark

Full list of author information is available at the end of the article
}

C C The Author(s). 2020 Open Access This article is licensed under a Creative Commons Attribution 4.0 International License, which permits use, sharing, adaptation, distribution and reproduction in any medium or format, as long as you give appropriate credit to the original author(s) and the source, provide a link to the Creative Commons licence, and indicate if changes were made. The images or other third party material in this article are included in the article's Creative Commons licence, unless indicated otherwise in a credit line to the material. If material is not included in the article's Creative Commons licence and your intended use is not permitted by statutory regulation or exceeds the permitted use, you will need to obtain permission directly from the copyright holder. To view a copy of this licence, visit http://creativecommons.org/licenses/by/4.0/ The Creative Commons Public Domain Dedication waiver (http://creativecommons.org/publicdomain/zero/1.0/) applies to the data made available in this article, unless otherwise stated in a credit line to the data. 


\section{Introduction}

Post-traumatic headache (PTH) is a disabling headache disorder and a common sequela of mild traumatic brain injury (TBI), [1]. The Third Edition of the International Classification of Headache Disorders (ICHD-3) defines PTH as a secondary headache disorder attributed to either mild TBI or moderate to severe TBI [2]. PTH is characterized by onset of headache within 7 days of mild TBI and, if the headache persists for at least 3 months, the term persistent PTH is used [2].

While ICHD-3 conceptualizes persistent PTH as a secondary headache disorder, the definition and diagnosis remains controversial and some contest that persistent PTH is 'trauma-triggered migraine [3]. This is supported by the observation that persistent PTH most often mimics a migraine-like headache phenotype in clinicbased studies [4, 5], leading to use of the term 'posttraumatic migraine' [6]. Current therapeutic strategies for persistent PTH includes acute and preventive medications used for the treatment of migraine [4, 7], with only some supportive evidence of this approach from open-label studies in individuals with persistent PTH [8, 9]. It could be speculated that persistent PTH sometimes represents migraine unmasked by mild TBI [10].

As a step towards clarifying the relationship of persistent PTH to migraine, we performed a 28-day prospective diary study in patients with persistent PTH who had at least eight monthly headache days of moderate to severe intensity. Daily diary entries were used to classify each headache day by the phenotype it most mimics. Furthermore, we assigned a final headache phenotype diagnosis based on diary review, without consideration of the ICHD-3 criterion for migraine and tension-type headache $(\mathrm{TTH})$ which states "not better accounted for by another ICHD-3 diagnosis" [2]. The aim was to characterize the range of headache phenotypes in individuals with persistent PTH and shed light on the similarities and differences between persistent $\mathrm{PTH}$ and migraine.

\section{Methods}

\section{Overview}

This study was approved by the Regional Health Research Ethics Committee of the Capital Region of Denmark (identifier: H-18050498), the Danish Medicines Agency (identifier: 2018-1104), and the Danish Data Protection Agency (identifier: VD-2019-20). Written informed consent was obtained from each participant before any study procedures or assessments were performed. The study was conducted in accordance with the Declaration of Helsinki [11].

Patients were recruited from an open-label trial set at the Danish Headache Center, Rigshospitalet Glostrup, Denmark [8]. Enrollment took place from April 2019 to
August 2019. At the screening visit, site investigators performed an in-person semi-structured interview to record data on demographics, medical history, and full clinical course [8]. Patients were then enrolled in a 28day paper diary study with daily entries and trained by the site investigators on completion of their diary.

Eligible patients included men or women aged 18 to 65 years with personal history of persistent headache attributed to mild TBI, in accordance with the ICHD-3 [2]. While ICHD-3 criteria defines persistent PTH by duration of headache for at least 3 months after mild TBI, we only included patients who had a history of persistent PTH for more than 12 months after mild TBI. Eligible patients were also required to report at least eight monthly headache days of moderate to severe intensity at the screening visit. Exclusion criteria for the diary study included 1) any personal history of primary headache disorder, except infrequent TTH pre-trauma, 2) any personal history of whiplash injury, and 3) medication-overuse headache, as defined by ICHD-3 [2]. To further ensure study eligibility, medical records were reviewed to cross-check for any formal headache diagnosis code. The full list of exclusion criteria has been published elsewhere [8].

\section{Measures and definitions}

A 28-day diagnostic headache diary was used to prospectively collect information on headache characteristics, associated symptoms, and use of acute medication. Patients were instructed to answer a daily set of questions at the end of each day and had been informed at the screening visit that the recall period was defined as the past $24 \mathrm{~h}$.

- A headache day of moderate to severe intensity was defined as any day with headache of moderate to severe intensity that lasted at least $4 \mathrm{~h}$.

- A migraine-like day was defined as any day with headache that lasted at least $4 \mathrm{~h}$ and fulfilled the ICHD-3 criteria for migraine without aura, migraine with aura, or probable migraine (2). Of note, patients were assigned with a TTH-like day if they met the criteria for probable migraine but also those for TTH. Furthermore, we also considered a migrainelike day to be defined by successful response to acute treatment with migraine-specific medication (i.e. triptan, ditan, gepant, ergot alkaloid).

- A TTH-like day was defined as any day with headache that lasted at least $30 \mathrm{~min}$ and fulfilled the ICHD-3 criteria for TTH [2].

Headache phenotypes were initially assigned based on diagnostic evaluation by trained medical students, while final headache phenotypes were assigned after diary 
review by two headache specialists (M.A. and H.W.S). Possible headache phenotypes included the following options:

- Episodic migraine-like, defined as headache occurring on $\geq 1$ day per month, which exclusively fulfills the criteria for a migraine-like day, but does not meet the requirement for a chronic migrainelike phenotype (see below).

- Chronic migraine-like, defined as headache occurring on $\geq 15$ days per month, which, on $\geq 8$ days per month fulfill the criteria for a migraine-like day.

- Episodic TTH-like, defined as headache occurring on 1-14 days per month, which exclusively fulfills the criteria for a TTH-like day.

- Chronic TTH-like, defined as headache occurring on $\geq 15$ days per month, which exclusively fulfills the criteria for a TTH-like day.

- Combined episodic migraine-like and episodic TTHlike, defined as headache occurring on 2-15 days per month, which, on $\geq 1$ day per month fulfills the criteria for a migraine-like day, and on $\geq 1$ day per month fulfills the criteria for a TTH-like day, but does not meet the requirement for a chronic migraine-like phenotype (see above).

- Combined episodic migraine-like and chronic TTHlike, defined as headache occurring on $>15$ days per month, which, on $\geq 15$ days per months fulfills the criteria for a TTH-like day, and on 1-7 days per months fulfills the criteria for a migraine-like day.

\section{Statistical analysis}

Outcomes were calculated based on headache diary entries for all patients who demonstrated at least $90 \%$ compliance throughout the 28-day diary period. For each patient, we calculated the following: 1) number of monthly headache days of any intensity, 2) number of monthly headache days of moderate to severe intensity, 3) proportion of monthly headache days of any intensity that met the criteria for a migraine-like day or a TTHlike day, and 4) proportion of monthly headache of moderate to severe intensity that met the criteria for a migraine-like day or a TTH-like day. In addition, we examined the proportion of patients whose monthly headache days of any intensity were $100 \%$ migraine-like, $\geq 75 \%$ migraine-like, $100 \%$ TTH-like, or $\geq 75 \%$ TTH-like. The latter calculations were also performed separately for monthly headache days of moderate to severe intensity. R statistical software version 3.6.0 was used to generate all data listing, summaries, and statistical analyses.

\section{Results}

A total of 64 patients with persistent PTH were included in this 4-week prospective diary study. The mean (SD) age was 34.0 (11.1) years, and the mean (SD) years of education was 14.8 (2.9). Of 64 patients, 49 (77\%) were females and 15 (23\%) were males. The mean (SD) number of months with headache attributed to mild TBI was 61.3 (60.0). Characteristics of the study population are summarized in Table 1 . The mean (SD) headache frequency was 26.3 (2.9) days per month, while the mean (SD) headache frequency of moderate to severe intensity was 19.7 (6.6) days per month. A positive family history of migraine was reported by $32 \%$ of patients. In terms of treatment patterns, 58 of 64 patients (91\%) reported current use of acute medication, with 30 patients (47\%) having current or previous use of triptans. Of the latter, 17 of 30 patients (57\%) reported insufficient pain relief. A history of preventive medication use was reported by 49 patients, of whom 27 (55\%) reported failure of at least two preventive medications. Current use of preventive medication was reported by 29 patients (45\%).

Two headache specialists (M.A. and H.W.S.) assigned final headache phenotype diagnosis based on review of the prospectively collected 28-day headache diary entries $(\kappa=1)$. The most common phenotype was chronic migraine-like $(n=47,73 \%)$ followed by a combined episodic migraine-like and chronic TTH-like $(n=9,14 \%)$ and pure chronic TTH-like $(n=8,13 \%)$, (Table 2). The mean (SD) headache frequency was 26.6 (2.4) days per month in patients with a chronic migraine-like phenotype, 24.6 (3.8) days per month in those with a combined episodic migraine-like and chronic TTH-like phenotype, and 26.6 (3.6) days per month in patients with a pure chronic TTH-like phenotype. In terms of headache days of moderate to severe intensity, the mean (SD) frequency was 19.9 (6.5) days per month in patients with a chronic migraine-like phenotype, 17.7 (7.0) days per month in those with a combined episodic migraine-like and chronic TTH-like phenotype, and 20.3 (5.9) days per month in patients with a pure chronic TTH-like phenotype. A positive family history of migraine was reported by $32 \%$ with a chronic migraine-like phenotype $(n=47)$, $44 \%$ with a combined episodic migraine-like and chronic TTH-like phenotype $(n=9)$, and $38 \%$ with a 'pure' chronic TTH-like phenotype $(n=8)$.

\section{Migraine-like days and TTH-like days}

After diary review (Table 3), 35 of 64 patients (55\%) had at least $75 \%$ overlap of monthly migraine-like days with monthly headache days. If considering only overlap of monthly migraine-like days with monthly headache days of moderate to severe intensity, 35 of 64 patients (55\%) had $100 \%$ overlap while 39 of $64(61 \%)$ at least $75 \%$ overlap.

Eight of 64 patients (13\%) had 100\% overlap of monthly TTH-like days with monthly headache days, whereas a corresponding overlap of at least $75 \%$ was 
Table 1 Characteristics of the Study Population

\begin{tabular}{|c|c|}
\hline Variable & Persistent PTH $(n=64)$ \\
\hline Age, mean (SD), y & $34.0(11.1)$ \\
\hline Male/Female, \% & $23 / 77$ \\
\hline Body Mass Index, mean (SD), kg/m² & $26.2(5.6)$ \\
\hline \multicolumn{2}{|l|}{ Current Employment Status, \% } \\
\hline Full-time employed & 22 \\
\hline Part-time employed & 53 \\
\hline Unemployed & 22 \\
\hline Retired & 3 \\
\hline \multicolumn{2}{|l|}{ Education } \\
\hline Years of education, mean (SD), y & $14.8(2.9)$ \\
\hline \multicolumn{2}{|l|}{ Level of education, \% } \\
\hline No completed education besides secondary school or high school & 17 \\
\hline Skilled labor & 28 \\
\hline Bachelor's degree & 27 \\
\hline Higher education & 28 \\
\hline \multicolumn{2}{|l|}{ Injury Cause, \% } \\
\hline Fall & 28 \\
\hline Motor vehicle collision & 27 \\
\hline Sports-related injury & 20 \\
\hline Violence/assault & 5 \\
\hline Other unintentional injury & 20 \\
\hline Months with Headache attributed to Mild TBI, mean (SD), months & $61.3(60.0)$ \\
\hline Family History of Migraine, $\%$ & 32 \\
\hline \multicolumn{2}{|l|}{ Headache Frequency, mean (SD) } \\
\hline Monthly headache days of any intensity & $26.3(2.9)$ \\
\hline Monthly headache days of moderate to severe intensity & $19.7(6.6)$ \\
\hline \multicolumn{2}{|l|}{ Acute Medication Use } \\
\hline Current Acute Medication Use, No. of subjects (\%) & $58(91)$ \\
\hline \multicolumn{2}{|l|}{ Triptans } \\
\hline Current or previous use, No. of subjects (\%) & $30(47)$ \\
\hline Lack of efficacy, No. of subjects (\%) & $17(57)$ \\
\hline \multicolumn{2}{|l|}{ Prevention Medication Use } \\
\hline History of Preventive Medication Use, No. of subjects & 49 \\
\hline No drug failures, $\%$ & 14 \\
\hline Failure of $\geq 1$ drug, $\%$ & 86 \\
\hline Failure of $\geq 2$ drugs, $\%$ & 55 \\
\hline Failure of $\geq 3$ drugs, $\%$ & 37 \\
\hline Failure of $\geq 4$ drugs, $\%$ & 20 \\
\hline Treatment Naïve, No. of subjects & 15 \\
\hline Current Preventive Medication Use, No. of subjects & 29 \\
\hline
\end{tabular}


Table 2 Characteristics of Study Population based on Headache Phenotype Diagnosis following Diary Review

\begin{tabular}{|c|c|c|c|}
\hline Variable & $\begin{array}{l}\text { Chronic Migraine-Like } \\
(n=47)\end{array}$ & $\begin{array}{l}\text { Episodic Migraine-Like combined with TTH- } \\
\text { Like }(n=9)\end{array}$ & $\begin{array}{l}\text { Chronic TTH-Like } \\
(n=8)\end{array}$ \\
\hline Age, mean (SD), y & $34.5(12.0)$ & $34.3(7.9)$ & $31.5(8.0)$ \\
\hline Male/Female, $\%$ & $19 / 81$ & $33 / 67$ & $37 / 63$ \\
\hline Body Mass Index, mean (SD), kg/m² & $26.1(5.6)$ & $24.6(3.8)$ & $24.9(4.0)$ \\
\hline \multicolumn{4}{|l|}{ Current Employment Status, No. of subjects (\%) } \\
\hline Full-time employed & $10(21)$ & $2(22)$ & $2(25)$ \\
\hline Part-time employed & $25(53)$ & $4(44)$ & $5(63)$ \\
\hline Unemployed & $11(23)$ & $2(22)$ & $1(13)$ \\
\hline Retired & $1(2)$ & $1(11)$ & $0(0)$ \\
\hline \multicolumn{4}{|l|}{ Education } \\
\hline Years of education, mean (SD), y & $14.3(2.6)$ & $16.3(3.2)$ & $15.9(3.3)$ \\
\hline \multicolumn{4}{|l|}{ Level of education, No. of subjects (\%) } \\
\hline Secondary school or high school & $10(21)$ & $0(0)$ & $1(13)$ \\
\hline Skilled labor & $13(28)$ & $4(44)$ & $1(13)$ \\
\hline Bachelor's degree & 11 (23) & $3(33)$ & $3(38)$ \\
\hline Higher education & $13(28)$ & $2(22)$ & $3(38)$ \\
\hline \multicolumn{4}{|l|}{ Injury Cause, No. of subjects, \% } \\
\hline Fall & $12(26)$ & $3(33)$ & $3(38)$ \\
\hline Motor vehicle collision & $14(30)$ & $2(22)$ & $1(13)$ \\
\hline Sports-related injury & $11(23)$ & $1(11)$ & $1(13)$ \\
\hline Violence/assault & $1(2)$ & $1(11)$ & $1(13)$ \\
\hline Other unintentional injury & $9(19)$ & $2(22)$ & $2(25)$ \\
\hline $\begin{array}{l}\text { Months with Headache attributed to Mild TBI, mean (SD), } \\
\text { months }\end{array}$ & $55.0(55.1)$ & $89.0(73.4)$ & $70.0(58.0)$ \\
\hline Family History of Migraine, No. of subjects (\%) & $15(32)$ & $4(44)$ & $3(38)$ \\
\hline \multicolumn{4}{|l|}{ Headache Frequency, mean (SD) } \\
\hline Monthly headache days of any intensity & $26.6(2.4)$ & $24.6(3.8)$ & $26.6(3.6)$ \\
\hline Monthly headache days of moderate to severe intensity & $19.9(6.5)$ & $17.7(7.0)$ & $20.3(5.9)$ \\
\hline \multicolumn{4}{|l|}{ Migraine-Like Frequency, mean (SD) } \\
\hline Monthly migraine-like days & $22.5(6.8)$ & $3.6(1.9)$ & - \\
\hline Monthly definite migraine-like days & $17.6(8.4)$ & $3.0(2.1)$ & - \\
\hline Monthly probable migraine-like days & $4.8(6.1)$ & $0.6(1.1)$ & - \\
\hline \multicolumn{4}{|l|}{ TTH-Like Frequency, mean (SD) } \\
\hline Monthly TTH-like days & $4.1(6.3)$ & $21.0(4.6)$ & $26.6(3.6)$ \\
\hline \multicolumn{4}{|l|}{ Acute Medication Use } \\
\hline Current Acute Medication Use, No. of subjects (\%) & $44(94)$ & $7(78)$ & $7(88)$ \\
\hline \multicolumn{4}{|l|}{ Triptans } \\
\hline Current or previous use, No. of subjects & 24 & 5 & 1 \\
\hline $\begin{array}{l}\text { Lack of efficacy in those with history of acute medication use, No. } \\
\text { of subjects (\%) }\end{array}$ & $13(54)$ & $3(60)$ & $1(100)$ \\
\hline \multicolumn{4}{|l|}{ Prevention Medication Use } \\
\hline History of Preventive Medication Use, No. of subjects (\%) & $36(77)$ & $7(78)$ & $6(75)$ \\
\hline No drug failures, No. of subjects (\%) & $4(11)$ & $2(29)$ & $1(17)$ \\
\hline Failure of $\geq 2$ drugs, No. of subjects (\%) & $20(56)$ & $5(71)$ & $2(33)$ \\
\hline Preventive Treatment Naïve, No. of subjects & $11(23)$ & $2(22)$ & $2(25)$ \\
\hline Current Preventive Medication Use, No. of subjects (\%) & $20(43)$ & $5(56)$ & $4(50)$ \\
\hline
\end{tabular}


Table 3 Summary of Study Outcomes

\begin{tabular}{lc}
\hline & Persistent PTH ( $\boldsymbol{n = 6 4 )}$ \\
\hline Headache Frequency during the 4-Week Diary Period, mean (SD) & $26.3(2.9)$ \\
Headache days of any intensity & $19.7(6.6)$ \\
Monthly Headache Days, No. of subjects (\%) & $24(38)$ \\
Monthly headache days of any intensity were exclusively migraine-like days & $35(55)$ \\
At least 75\% of monthly headache days of any intensity were migraine-like days & 8 (13) \\
Monthly headache days of any intensity were exclusively TTH-like days & $16(25)$ \\
At least 75\% of monthly headache days of any intensity were TTH-like days & $35(55)$ \\
Monthly Headache Days of Moderate to Severe Intensity, No. of subjects (\%) & $39(61)$ \\
Monthly headache days of moderate to severe intensity were exclusively migraine-like days & 8 (13) \\
At least 75\% of monthly headache days of moderate to severe intensity were migraine-like days & $15(23)$ \\
Monthly headache days of moderate to severe intensity were exclusively TH-like days & \\
At least 75\% of monthly headache days of moderate to severe intensity were TTH-like days &
\end{tabular}

found in 16 of 64 patients (25\%), (Table 3). If considering only overlap of monthly TTH-like days with monthly headache days of moderate to severe intensity, 9 of 64 (13\%) had $100 \%$ overlap while 15 of 64 (23\%) had at least $75 \%$ overlap.

\section{Reclassification of initial headache phenotype diagnosis based on diary review}

At the initial diagnostic evaluation, the most common phenotypes assigned were chronic migraine-like $(n=45$, $70 \%)$ followed by combined episodic migraine-like and chronic TTH-like $(n=11,17 \%)$, and pure chronic TTHlike $(n=8,13 \%)$, (Table 4$)$. After a diary review, the corresponding figures were $73 \%, 14 \%$, and $13 \%$. Ten of 64 patients had their initial headache phenotype diagnosis reclassified based on diary review (Table 4). Trained medical students were able to provide an accurate initial headache phenotype diagnosis in $84 \%$ of patients by retrospective assessment using a semi-structured interview.

\section{Discussion}

In this study of 64 patients with persistent PTH and at least eight monthly headache days of moderate to severe intensity, we collected prospective diary data to examine the overlap of monthly migraine-like days with monthly headache days. Taken together, we observed that most patients were assigned with a chronic migraine-like phenotype and monthly headache days more often overlapped with monthly migraine-like days than monthly TTH-like days. However, we did also find that some patients had 100\% overlap between monthly TTH-like days and monthly headache days and were, thus, assigned with a pure chronic TTH-like phenotype. At minimum, our findings establish that not all individuals with persistent PTH have a migraine-like phenotype. It should, furthermore, be emphasized that none of the included patients had a personal history of migraine.

Phenotyping of clinical features are important to establish a more comprehensive and symptom-based classification of PTH [12]. The ICHD-3 recommends phenotyping of a patient's clinical features using a headache diary when more than one headache type/subtype is suspected [2]. However, phenotyping of acute and persistent PTH has only been made with methods that are limited by recall bias, e.g. questionnaires and interviews [4, 13-17]. The present study provides the first diary data, in which clinical features of persistent PTH were collected daily. Future diary studies should include larger cohorts to identify subgroups of patients with PTH based on their headache phenotype (e.g. migraine-like, TTH-like) and personal history of primary headache disorder (e.g. pre-existing migraine).

A considerable limitation of the current ICHD-3 diagnostic criteria is its emphasis on expert opinion rather than scientific evidence, which is largely attributed to the scarcity of literature on PTH $[2,10,13]$. Currently the ICHD-3 criteria classifies persistent PTH as 'any headache', which develops within 7 days after TBI and persists for more than 3 months after its onset [2]. The implications are that clinicians are left without information on clinical features or frequency thresholds. Fortunately, there is an increasing number of observational clinic-based studies being published, in which headache phenotypes have been assigned. These studies have found that the clinical features of PTH often mimics a migraine-like or TTH-like phenotype but were limited by their method of assessment, i.e. questionnaires and interview [4, 13-17]. Nonetheless, their findings were confirmed in our prospective diary study, in which all 
Table 4 Reclassification of Initial Headache Phenotype Diagnosis based on Diary Review

\begin{tabular}{|c|c|c|c|c|}
\hline \multirow{2}{*}{$\begin{array}{l}\text { Initial Headache } \\
\text { Phenotype based on } \\
\text { Semi-structured Inter- } \\
\text { view, No. of subjects }\end{array}$} & \multicolumn{3}{|c|}{ Final Headache Phenotype based on Diary Review, No. of subjects } & \multirow{2}{*}{$\begin{array}{l}\text { Total, } \\
\text { No. of } \\
\text { subjects } \\
\text { (\%) }\end{array}$} \\
\hline & CM-Like & EM-Like + CTTH-Like & CTTH-Like & \\
\hline CM-Like & 42 & 2 & 1 & $45(70)$ \\
\hline EM-Like + CTTH-Like & 2 & 7 & 2 & $11(17)$ \\
\hline CTTH-Like & 3 & 0 & 5 & $8(13)$ \\
\hline Total, No. of subjects (\%) & $47(73)$ & $9(14)$ & $8(13)$ & $64(100)$ \\
\hline
\end{tabular}

No. Number, CM-Like Chronic Migraine-Like, EM-Like Episodic Migraine-Like, CTTH-Like Chronic TTH-Like

headache days reported could be classified as either migraine-like days or TTH-like days. It remains unknown whether treatment strategies guided by assigned headache phenotypes holds any value [9]. Nonetheless, it would be interesting to explore this issue further in the future.

7An important implication of the present diary study is that some individuals with persistent PTH do not experience migraine-like headache, which confirms previous reports based on phenotype assessment by questionnaire or interview [4,13-17]. Thus, it would be erroneous to continue the discussion of whether persistent PTH is trauma-triggered migraine. At the most, it could be speculated that migraine was unmasked by mild TBI in those with a migraine-like phenotype. If so, biological underpinnings should be similar between the two groups. This does not seem to be the case based on findings from magnetic resonance imaging (MRI) studies, in which differences in brain structure and function were found when comparing patients with persistent PTH to patients with migraine [18-21]. It should be mentioned that the included patients with persistent PTH were mostly assigned with a migraine-like phenotype, although they did not have a personal history of migraine [18-21]. These data are suggestive of persistent PTH not being trauma-triggered migraine $[10,21]$. Furthermore, one recent study explored plasma levels of calcitonin gene-related peptide (CGRP) in individuals with persistent PTH [22]. The authors found that CGRP plasma levels were lower in 100 individuals with persistent PTH when compared with 100 age- and gendermatched healthy non-headache controls. This observation is interesting given that elevated plasma/serum levels of CGRP have recently been proposed as a biomarker candidate for the ictal phase of migraine [2325]. It should also be mentioned that numerous randomized clinical trials (RCTs) have demonstrated therapeutic benefit of monoclonal antibodies (mAbs) targeting CGRP or its receptor for migraine prevention [26-33]. Although there is no comparable RCT data, one 12week open-label trial did find that $28 \%$ of individuals with persistent $\mathrm{PTH}$ experienced at $50 \%$ reduction in monthly headache days of moderate to severe intensity after treatment with a mAb targeting the CGRP receptor (erenumab), [8]. Taken together, more research is needed to ascertain similarities and differences between persistent PTH and migraine [34-38]. Such investigations should also assess the role of family history of migraine, response to migraine-specific medication, as well as involvement of biomarkers associated with migraine (e.g. genetic, provocation, biochemistry, imaging).

\section{Limitations}

A limitation of the present study was the modest sample size and a study population which only included patients with persistent PTH who experienced at least eight monthly headache days of moderate to severe intensity. Thus, our study population is skewed towards reflecting more adversely affected individuals with persistent PTH in the general population. However, this was intended as we aimed to assess the overlap of monthly migraine-like days with monthly headache days in patients most burdened by persistent PTH. Another limitation was that our outcomes were assessed by entries in a paper format diary, which does not allow monitoring of daily entries. However, the included patients had been informed at the screening visit that the recall period was defined as the past $24 \mathrm{~h}$.

\section{Conclusions}

A migraine-like phenotype is most common in patients most adversely affected by persistent $\mathrm{PTH}$, although a substantial minority of patients do have a pure TTH-like phenotype. These findings - at minimum - dismiss the notion that persistent PTH is trauma-triggered migraine. Further studies are needed to ascertain similarities and differences of biological underpinnings between persistent PTH and migraine.

\section{Abbreviations}

PTH: Post-traumatic headache; TBI: Traumatic brain injury; ICHD-

3: International Classification of Headache Disorders, Third Edition; TTH: Tension-type headache; CGRP: Calcitonin gene-related peptide; RCT: Randomized clinical trial; mAbs: Monoclonal antibodies

Acknowledgments

Not applicable. 


\section{Authors' contributions}

Håkan Ashina: Study concept and design, acquisition of data, analysis (including statistical analyses) and interpretation, drafting the manuscript. Afrim Iljazi: Acquisition of data and critical revision of the manuscript for important intellectual content. Faisal M. Amin: Acquisition of data and critical revision of the manuscript for important intellectual content. Messoud Ashina: Study concept and design, and critical revision of the manuscript for important intellectual content. Richard B. Lipton: Study concept and design, and critical revision of the manuscript for important intellectual content. Henrik W. Schytz: Study concept and design, and critical revision of the manuscript for important intellectual content. The author(s) read and approved the final manuscript.

\section{Funding}

The study was supported by an investigator-initiated research grant from Novartis Healthcare A/S.

\section{Availability of data and materials}

Qualified researchers can request access to patient-level data and related study documents, including the study protocol. Patient-level data will be deidentified and study documents will be redacted to protect the privacy of study participants.

\section{Ethics approval and consent to participate}

Informed written consent was obtained from each patient before conducting any study-related procedures. The study protocol was reviewed by the Regional Health Research Ethics Committee of the Capital Region of Denmark (identifier: $\mathrm{H}-18050498$ ).

\section{Consent for publication}

Not applicable.

\section{Competing interests}

The authors declared the following potential conflicts of interest with respect to the research, authorship and/or publication of this article: Dr. Faisal M. Amin has received personal fees from Eli Lilly, Novartis, and Teva. Dr. Faisal M. Amin is currently a principal investigator of clinical trials funded by Novartis and Teva. Dr. Faisal M. Amin is an associate editor of Headache Medicine. Dr. Messoud Ashina has received personal fees from Alder BioPharmaceuticals, Allergan, Amgen, Eli Lilly, Novartis, and Teva. Dr. Messoud Ashina has been or is currently a principal investigator of clinical trials funded by Alder BioPharmaceuticals, Amgen, electroCore, Novartis, and Teva. Dr. Messoud Ashina is as an associate editor of Cephalalgia, Headache, and the Journal of Headache and Pain. Dr. Messoud Ashina reports research grants from the Lundbeck Foundation, Novo Nordisk Foundation, and Research Foundation of the Capital Region of Denmark. Dr. Richard B. Lipton is the Edwin S. Lowe Professor of Neurology at the Albert Einstein College of Medicine in New York. He receives research support from the $\mathrm{NIH}$ : 2PO1 AG003949 (Multiple Principal Investigator), 5 U10 NS077308 (Principal Investigator), RO1 NS082432 (Investigator), 1RF1 AG057531 (Site Principal Investigator), RF1 AG054548 (Investigator), 1RO1 AG048642 (Investigator), R56 AG057548 (Investigator), K23 NS09610 (Mentor), K23AG049466 (Mentor), and 1K01AG054700 (Mentor). He also receives support from the Migraine Research Foundation and the National Headache Foundation. He serves on the editorial board of Neurology, senior advisor to Headache, and associate editor to Cephalalgia. He has reviewed for the NIA and NINDS; holds stock options in eNeura Therapeutics and Biohaven Holdings; and serves as consultant, advisory board member, or has received honoraria from the American Academy of Neurology, Alder, Allergan, American Headache Society, Amgen, Autonomic Technologies, Avanir, Biohaven, Biovision, Boston Scientific, Dr. Reddy's, electroCore, Eli Lilly, eNeura Therapeutics, GlaxoSmithKline, Merc k, Pernix, Pfizer, Supernus, Teva, Trigemina, Vector, and Vedanta. He receives royalties from Wolff's Headache, 7th and 8th Edition, Oxford Press University, 2009, Wiley and Informa. Dr. Henrik W. Schytz has received speaking fees from Novartis, Eli Lilly, and Teva. Dr. Henrik W. Schytz has received a research grant from Novartis. The other authors declare no conflicts of interest.

\section{Author details}

'Danish Headache Center, Department of Neurology, Rigshospitalet Glostrup, Faculty of Health and Medical Sciences, University of Copenhagen, Valdemar
Hansen Vej 5, Glostrup, DK-2600 Copenhagen, Denmark. ${ }^{2}$ Department of Neurology, Albert Einstein College of Medicine, Bronx, NY, USA. ${ }^{3}$ Montefiore Medical Center, Bronx, NY, USA.

Received: 8 September 2020 Accepted: 10 November 2020

Published online: 19 November 2020

\section{References}

1. Nampiaparampil DE (2008) Prevalence of chronic pain after traumatic brain injury: a systematic review. JAMA 300:711-719

2. Headache Classification Committee of the International Headache Society (2018) The international classification of headache disorders (ICHD), 3rd edition. Cephalalgia 38:1-211

3. Haas DC, Lourie H (1988) Trauma-triggered migraine: an explanation for common neurological attacks after mild head injury. Review of the literature. J Neurosurg 68:181-188

4. Ashina H, Iljazi A, Al-Khazali HM et al (2020) Persistent post-traumatic headache attributed to mild traumatic brain injury: deep phenotyping and treatment patterns. Cephalalgia 40:554-564

5. Theeler B, Lucas S, Riechers RG II et al (2013) Post-traumatic headaches in civilians and military personnel: a comparative, clinical review. Headache 53 $881-900$

6. Weiss HD, Stern BJ, Goldberg J (1991) Post-traumatic migraine: chronic migraine precipitated by minor head or neck trauma. Headache 31:451-456

7. Lucas S (2015) Posttraumatic headache: clinical characterization and management. Curr Pain Headache Rep 19:48

8. Ashina H, Iljazi A, Al-Khazali HM et al (2020) Efficacy, tolerability, and safety of erenumab for the preventive treatment of persistent post-traumatic headache attributed to mild traumatic brain injury: an open-label study. J Headache Pain 21:62

9. Larsen EL, Ashina H, lljazi A et al (2019) Acute and preventive pharmacological treatment of post-traumatic headache: a systematic review. J Headache Pain 20:98

10. Ashina H, Porreca F, Anderson T et al (2019) Post-traumatic headache: epidemiology and pathophysiological insights. Nat Rev Neurol 15:607-617

11. World Medical Association (2013) World medical association declaration of Helsinki: ethical principles for medical research involving human subjects. JAMA 310:2191-2194

12. Lucas S, Ahn AH (2018) Posttraumatic headache: classification by symptombased clinical profiles. Headache 58:873-882

13. Lieba-Samal $D$, Platzer $P$, Seidel $S$ et al (2011) Characteristics of acute posttraumatic headache following mild head injury. Cephalalgia 31:16181626

14. Hoffman JM, Lucas S, Dikmen S et al (2011) Natural history of headache after traumatic brain injury. J Neurotrauma 28:1719-1725

15. Lucas S, Hoffman JM, Bell KR et al (2012) Characterization of headache after traumatic brain injury. Cephalalgia 32:600-606

16. Lucas S, Hoffman JM, Bell KR et al (2014) A prospective study of prevalence and characterization of headache following mild traumatic brain injury. Cephalalgia 34:93-102

17. Stacey A, Lucas S, Dikmen S et al (2017) Natural history of headache five years after traumatic brain injury. J Neurotrauma 34:1558-1564

18. Schwedt TJ, Chong CD, Peplinski J et al (2017) Persistent post-traumatic headache vs. migraine: an MRI study demonstrating differences in brain structure. J Headache Pain 18:87

19. Chong CD, Peplinski J, Berisha V et al (2019) Differences in fibertract profiles between patients with migraine and those with persistent post-traumatic headache. Cephalalgia 39:1121-1133

20. Dumkrieger G, Chong CD, Ross K et al (2019) Static and dynamic functional connectivity differences between migraine and persistent post-traumatic headache: a resting-state magnetic resonance imaging study. Cephalalgia 39:1366-1381

21. Schwedt TJ (2019) Structural and functional brain alterations in posttraumatic headache attributed to mild traumatic brain injury: a narrative review. Front Neurol 10:615

22. Ashina $\mathrm{H}$, Al-Khazali HM, Iljazi A et al (2020) Low plasma levels of calcitonin gene-related peptide in persistent post-traumatic headache attributed to mild traumatic brain injury. Cephalalgia online ahead of print [20 July 2020]. https://doi.org/10.1177/0333102420941115 
23. Davis KD, Aghaeepour N, Ahn AH et al (2020) Discovery and validation of biomarkers to aid the development of safe and effective pain therapeutics: challenges and opportunities. Nat Rev Neurol 16:381-400

24. Goadsby PJ, Edvinsson L, Ekman R (1990) Vasoactive peptide release in the extracerebral circulation of humans during migraine headache. Ann Neurol 28:183-187

25. Gallai V, Sarchielli P, Floridi A et al (1995) Vasoactive peptide levels in the plasma of young migraine patients with and without aura assessed both interictally and ictally. Cephalalgia 15:384-390

26. Goadsby PJ, Reuter U, Hallström Y et al (2017) A controlled trial of Erenumab for episodic migraine. N Engl J Med 377:2123-2132

27. Reuter U, Goadsby PJ, Lanteri-Minet M et al (2018) Efficacy and tolerability of erenumab in patients with episodic migraine in whom two-to-four previous preventive treatments were unsuccessful: a randomised, doubleblind, placebo-controlled, phase 3b study. Lancet. 392:2280-2287

28. Silberstein SD, Dodick DW, Bigal ME et al (2017) Fremanezumab for the preventive treatment of chronic migraine. N Engl J Med 377:2113-2122

29. Ferrari MD, Diener HC, Ning $X$ et al (2019) Fremanezumab versus placebo for migraine prevention in patients with documented failure to up to four migraine preventive medication classes (FOCUS): a randomised, doubleblind, placebo-controlled, phase 3b trial. Lancet 394:1030-1040

30. Stauffer VL, Dodick DW, Zhang Q et al (2018) Evaluation of Galcanezumab for the prevention of episodic migraine: the EVOLVE-1 randomized clinical trial. JAMA Neurol 75:1080-1088

31. Detke HC, Goadsby PJ, Wang S et al (2018) Galcanezumab in chronic migraine: the randomized, double-blind, placebo-controlled REGAIN study. Neurology 91:e2211-e2221

32. Ashina M, Saper J, Cady R et al (2020) Eptinezumab in episodic migraine: a randomized, double-blind, placebo-controlled study (PROMISE-1). Cephalalgia 40:241-254

33. Lipton RB, Goadsby PJ, Smith J et al (2020) Efficacy and safety of eptinezumab in patients with chronic migraine: PROMISE-2. Neurology 94: e1365-e1377

34. Benemei S, Labastida-Ramírez A, Abramova E et al (2020) Persistent posttraumatic headache: a migrainous loop or not? The preclinical evidence. J Headache Pain 21:90

35. Labastida-Ramírez A, Benemei S, Albanese M et al (2020) Persistent posttraumatic headache: a migrainous loop or not? The clinical evidence. J Headache Pain 21:55

36. Capi M, Pomes LM, Andolina G et al (2020) Persistent post-traumatic headache and migraine: pre-clinical comparisons. Int J Environ Res Public Health 17:2585

37. Ashina M. Migraine. N Engl J Med. 2020;383:1866-76.

38. Mitsikostas DD, Moskowitz MA. Making headway - a role for CGRP in posttraumatic headache. Nat Rev Neurol. 2020. https://doi.org/10.1038/s41582020-00431-2.

\section{Publisher's Note}

Springer Nature remains neutral with regard to jurisdictional claims in published maps and institutional affiliations.

Ready to submit your research? Choose BMC and benefit from:

- fast, convenient online submission

- thorough peer review by experienced researchers in your field

- rapid publication on acceptance

- support for research data, including large and complex data types

- gold Open Access which fosters wider collaboration and increased citations

- maximum visibility for your research: over $100 \mathrm{M}$ website views per year

At $\mathrm{BMC}$, research is always in progress.

Learn more biomedcentral.com/submissions 